\title{
Reciprocating Compressor 1D Thermofluid Dynamic Simulation: Problems and Comparison with Experimental Data
}

\author{
A. Gimelli, ${ }^{1}$ A. Rapicano, ${ }^{1}$ F. Barba, ${ }^{2}$ and O. Pennacchia ${ }^{1}$ \\ ${ }^{1}$ Dipartimento di Meccanica ed Energetica (DiME), Università Degli Studi di Napoli Federico II, 80125 Napoli, Italy \\ ${ }^{2}$ Facoltà di Ingegneria, Università Degli Studi di Napoli Federico II, 80125 Napoli, Italy
}

Correspondence should be addressed to A. Gimelli, gimelli@unina.it

Received 9 February 2012; Revised 27 March 2012; Accepted 31 March 2012

Academic Editor: Ryo Amano

Copyright (C) 2012 A. Gimelli et al. This is an open access article distributed under the Creative Commons Attribution License, which permits unrestricted use, distribution, and reproduction in any medium, provided the original work is properly cited.

\begin{abstract}
The authors here extend a 0D-1D thermofluid dynamic simulation approach to describe the phenomena internal to the volumetric machines, reproducing pressure waves' propagation in the ducts. This paper reports the first analysis of these phenomena in a reciprocating compressor. The first part presents a detailed experimental analysis of an open-type reciprocating compressor equipped with internal sensors. The second part describes a 0D-1D thermofluid dynamic simulation of the compressor. Comparison of computed and measured values of discharge mass flow rate shows a good agreement between results for compression ratio $\beta<5$. Then, to improve the model fitting at higher pressures, a new scheme has been developed to predict the blow-by through the ring pack volumes. This model is based on a series of volumes and links which simulate the rings' motions inside the grooves, while the ring dynamics are imposed using data from the literature about blow-by in internal combustion engines. The validation is obtained comparing experimental and computing data of the two cylinder engine blowby. After the validation, a new comparison of mass flow rate on the compressor shows a better fitting of the curves at higher compression ratio.
\end{abstract}

\section{Introduction}

1.1. Background. Volumetric compressors, alternative and rotary, represent an element of large utilities in the domestic field as in industrial applications, in particular, the refrigeration cycle $[1,2]$. In today's world, energy optimization is of the utmost importance, therefore, it is crucial to find tools which can support the planning phase to increase the efficiency of these machines. Imagine, that approximately $11 \%$ of the produced electrical energy today is destined to power domestic refrigeration [3]. Reported in this paper is a method for the simulation of $0 \mathrm{D}-1 \mathrm{D}$ fluid-dynamic phenomena which occurs in the volumetric machines, noting the results from a study of problems linked to volumetric reciprocating engine, that in the future will be extended also to rotary. For years, the most widely used and reliable approach was the empirical one [4], because of the complexity of physical phenomena that takes place in the thermal machines. This occurs because the functioning at full performance of a volumetric compressor is not stationary but periodic; equations that govern the mass transport in the pipelines are not linear, so they cannot be resolved through an analytic method. However, the development of computing potential and of theoreticalnumeric methods suggest a new approach, based on the use of fluid-dynamic models. These models are divided into a different kind of sophistication. Depending on the user's needs, there are semiempirical models, whose equations are studied on the results of a focused experimentation [3], simplified analytics models, quite common in the industrial field [2] as they describe the compressor overall. Finally, there are more thorough models [4-6], governed by differential not stationary equations linked to the individual volumes in which the machine is divided. The thorough models are very important in the research field as they allow us to study many phenomena which take place inside the machine. For example, it was proved that the pressure waves that travel through the discharge pipes, governed also by the dynamics of automatic or controlled valves $[7,8]$, are a major source of noise radiation from reciprocating compressors. The detection of these parameters which govern these pulsations can lead to the development of instruments which could 


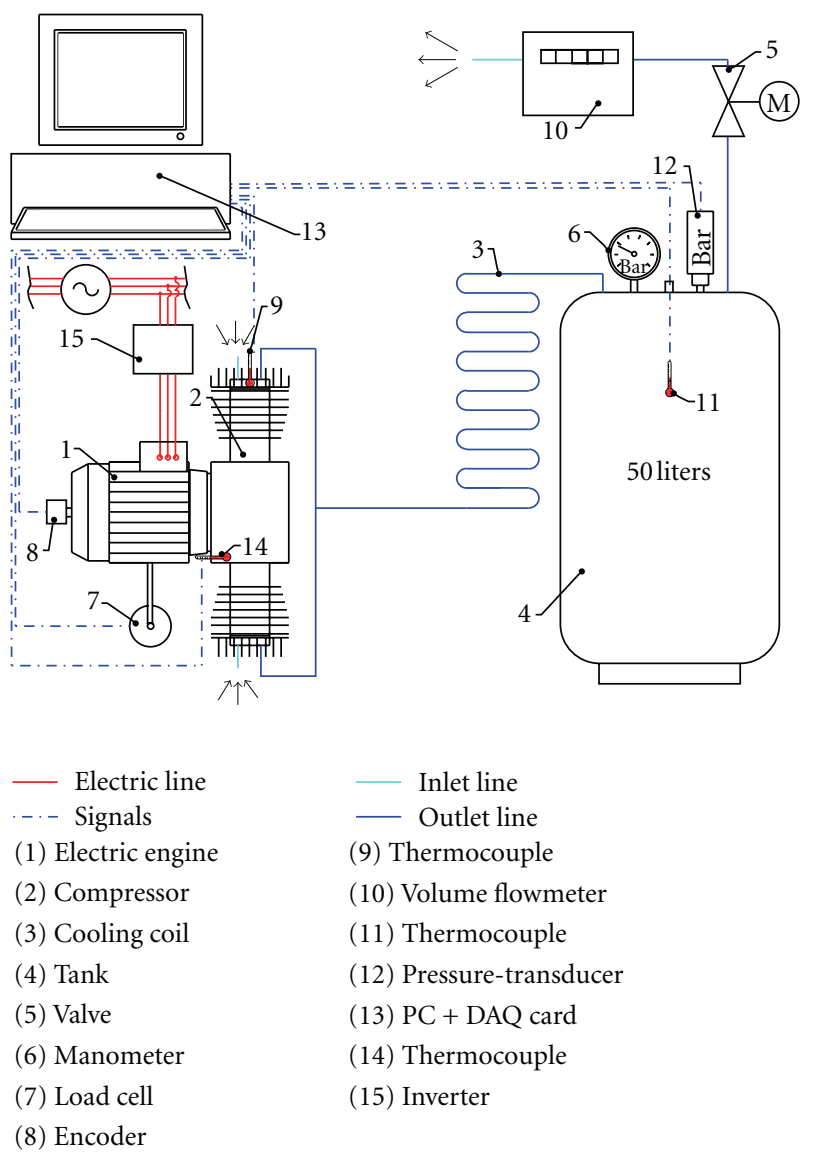

Figure 1: Test bed layout.

reduce this noise radiation. Thanks to this kind of approach, a substantial reduction of cost and development time can be obtained, allowing in just a short time a sensitivity analysis to the many functional and structural parameters showing the best solutions to be tested experimentally.

1.2. Targets of the Thermofluid Dynamic Simulation. In this perspective, during the last two decades [6,9-25], a 0D1D fluid-dynamic simulation software was developed at DiME for the alternative internal combustion engine. Based on the resolution of flow equations in the pipes out of the cylinders, adopting a one-dimensional schematization, while the capacity in variable volume condition (the cylinder) is resolved through a zero-dimensional approach, it was considered that variables do not change on a spatial coordinate, but they are only a function of time. Through the use of this tool, in the first part of this work, a new scheme for the simulation of a V-twin compressor was proposed, starting from the knowledge of the construction features of the machine. In particular, the layout of a test bed was reproduced, on which the stationary tests were carried out, having provided the average values of pressure, temperature, and airflow at the outlet. The comparison between the experimental and simulated values has allowed a first check of the results to be obtained. Then, the "blow-by" has been studied, which is the phenomenon of gas leakage

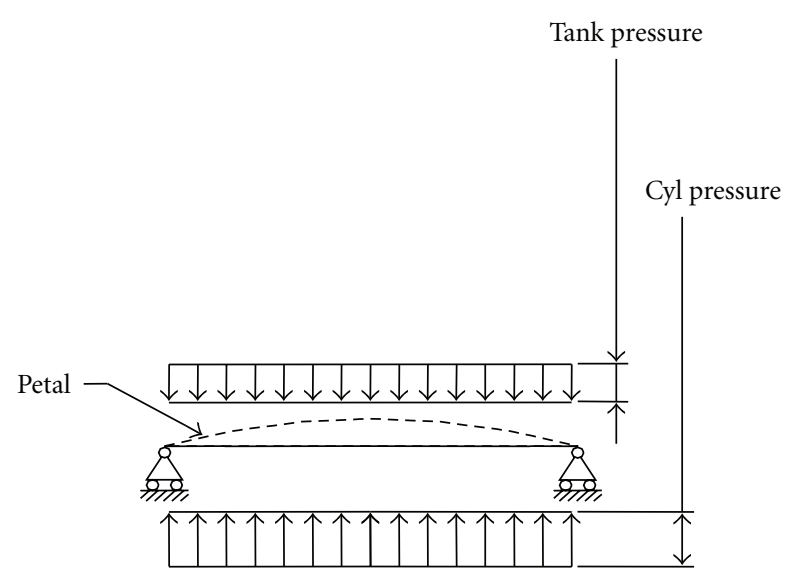

(a)
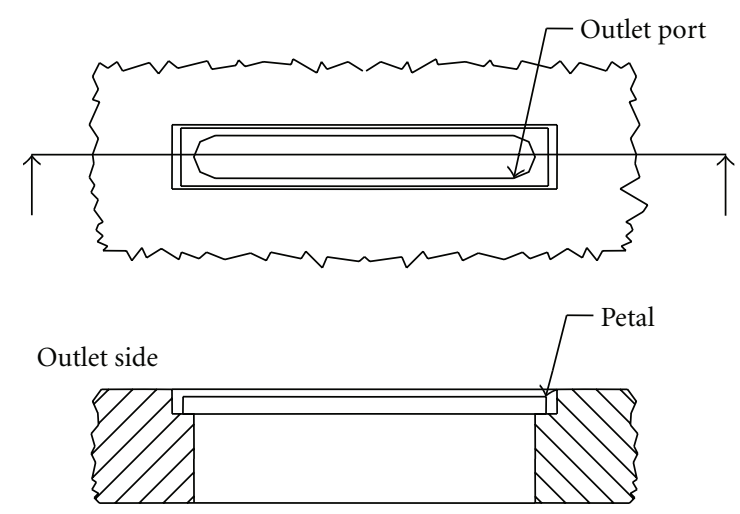

Cylinder side

(b)

Figure 2: (a) Reed valve deflection, (b) outlet valve.
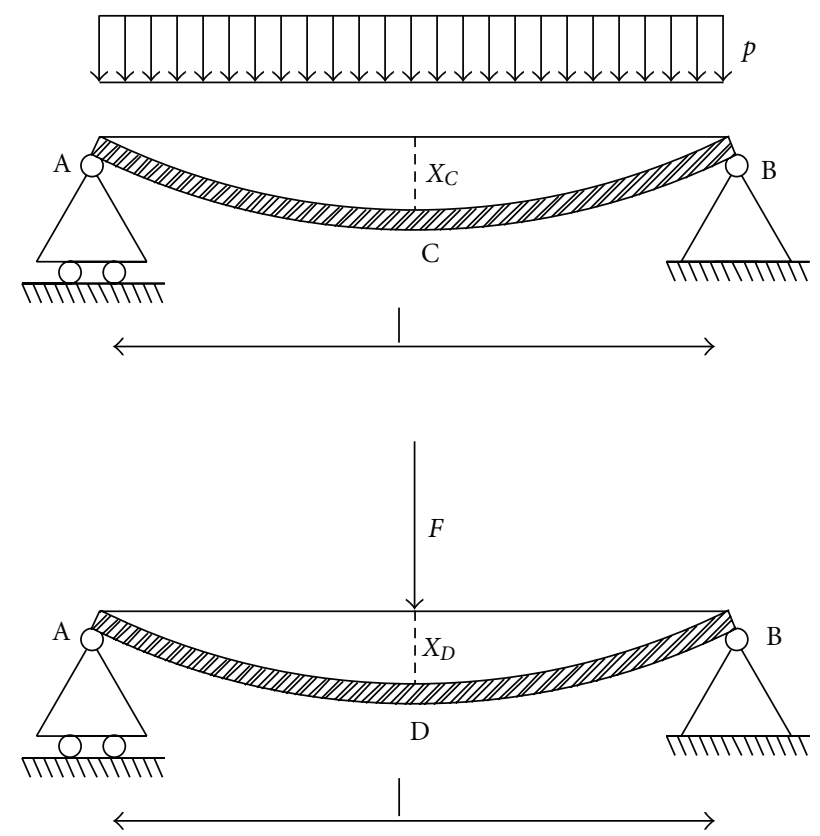

FIGURE 3: Deflection of a simply supported beam under uniformly distributed and concentrated load. 


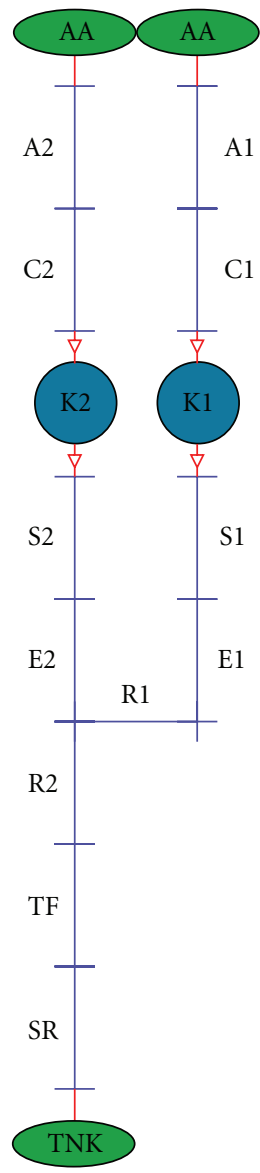

\footnotetext{
External environment volume

Zero-dimensional element with variable volume Mono-dimensional element

$\rightarrow$ Valve link

_ Constant cross section link
}

FIGURE 4: Geometric layout of the compressor.

that takes place between the compression chamber and the crankcase as a result of the clearance between piston, cylinder liner, and rings. To this end, starting from [8] and from the current knowledge about the rings dynamic inside the internal combustion engine [26], a model for the simulation of "blow-by" inside the compressors has been implemented. The targets to achieve here were as follows:

(i) to improve the predictability of the $0 \mathrm{D}-1 \mathrm{D}$ model introducing a scheme which considers the leakage in cylinders in the volumetric compressors,

(ii) to provide an indicative framework of the fluid dynamic phenomena which take place inside the piston sealing system, also in relation to the segments dynamic,

(iii) to suggest a new approach to volumetric machine simulation taking into account the leakage phenomena that is not negligible, especially in the rotary machines.

\section{Experimental Setup}

The compressor which is the object of this theoretical and experimental analysis is a FIAC V254, a twin reciprocating $\mathrm{v}$-unit with its total displacement being $165 \mathrm{~cm}^{3}$. Figure 1 represents the layout of the equipment with which the tests were made. The compressor (2) is moved by a threephase asynchronous electric motor (1) of $2.2 \mathrm{~kW}$ lead to the nominal speed of $1500 \mathrm{rpm}$ through an inverter (15). Motor and compressor are connected by a flexible coupling. The compressor feeds a 50 liter tank (4).

A cooling coil (3) is placed between the compressor and the tank to cool down the air mass flow before it reaches the tank. Downstream of the heat exchanger is placed in a manual lamination valve (5) which is necessary both to perform constant pressure tests and to empty the tank at the end of the session. After which the lamination valve is placed in a volumetric flowmeter (10). In Table 1, data are collected in 10 stationary tests, with each test featuring a constant pressure in the tank [27].

\section{Numerical Models: The Code}

A computer code recently developed by DiME researchers has been employed, for the evaluation of component matching and estimation of the in-cylinder and in-pipe processes [6,9-25]. The code, being of a modular type, can easily handle different engine configurations (i.e., single or multicylinder compression-ignition engines [13-15], twostroke spark-ignition engines $[6,10,11,19,24]$, such as four strokes, either aspirated or supercharged $[18,20,22,23]$, etc.). Moreover, three-dimensional models in the cylinder have been integrated such as the KIVA [15-17, 22, 24], to evaluate the $3 \mathrm{D}$ phenomena in a transient state.

$O D$ Model. A filling and emptying technique is adopted for calculating the time-varying conditions inside variable volume devices (i.e., cylinders, displacement compressors, etc.) The general formulation of the governing equations for mass and energy balance within a control volume is shown below. Particular care was paid to the determination of gas properties: The mass equation has been split into three equations (1), (2), and (3), which, respectively, take into account the total mass balance and those of residual and fuel mass fractions:

$$
\begin{gathered}
\dot{m}=\dot{m}_{f}^{\mathrm{inj}}+\sum_{i} \dot{m}_{i}^{\mathrm{vlv}}, \quad \dot{m}=\dot{m}_{a}+\dot{m}_{r}+\dot{m}_{f}, \\
\dot{m}_{r}=\sum_{i} \dot{m}_{r, i}^{\mathrm{vlv}}+\dot{m}_{f}^{\mathrm{cmb}}\left(\frac{1+f_{\mathrm{st}}}{f_{\mathrm{st}}}\right), \\
\dot{x}_{r}=\frac{1}{m}\left(\dot{m}_{r}-x_{r} \dot{m}\right), \quad x_{r}=\frac{m_{r}}{m}, \\
\dot{m}_{f}=\dot{m}_{f}^{\mathrm{inj}}+\sum_{i} \dot{m}_{f, i}^{\mathrm{vlv}}-\dot{m}_{f}^{\mathrm{cmb}}, \\
\dot{x}_{f}=\frac{1}{m}\left(\dot{m}_{f}-x_{f} \dot{m}\right), \quad x_{f}=\frac{m_{f}}{m} .
\end{gathered}
$$


TABLE 1: Experimental data.

\begin{tabular}{|c|c|c|c|c|c|}
\hline Test number & $p_{\text {amb }}($ bar $)$ & $T_{\mathrm{amb}}(\mathrm{K})$ & $n(\mathrm{rpm})$ & $P_{\text {tnk }}($ bar $)$ & $T_{\text {oil }}(\mathrm{K})$ \\
\hline 9 & 1.017 & 299 & 1487 & 9.998 & 340 \\
\hline 8 & 1.017 & 299 & 1498 & 9.019 & 331 \\
\hline 7 & 1.017 & 299 & 1495 & 8.010 & 340 \\
\hline 6 & 1.017 & 299 & 1493 & 7.024 & 337 \\
\hline 5 & 1.017 & 299 & 1494 & 6.019 & 335 \\
\hline 4 & 1.017 & 299 & 1504 & 5.045 & 331 \\
\hline 3 & 1.017 & 299 & 1497 & 3.994 & 328 \\
\hline 2 & 1.017 & 299 & 1495 & 2.998 & 324 \\
\hline 1 & 1.017 & 299 & 1506 & 2.017 & 321 \\
\hline 0 & 1.017 & 299 & 1506 & 1.199 & 318 \\
\hline
\end{tabular}

The energy equation was properly rearranged with a correct gas mixture property calculation:

$$
\dot{T}=\frac{(1 / m)\left[-p \dot{V}-\dot{Q}_{w}+\dot{m}_{f}^{\mathrm{inj}} h_{f}^{(o)}+\sum_{i} \dot{m}_{i} h_{i}^{\mathrm{vlv}}-e \dot{m}-m\left(\partial e / \partial x_{r}\right) \dot{x}_{r}\right]-\left(\left(p / A_{p}\right)(\partial e / \partial p)\right)\left(\dot{m} / m-\dot{V} / V+\left((1 / R)\left(\partial R / \partial x_{r}\right)\right) \dot{x}_{r}\right)}{c_{v}+(p / T)\left(A_{T} / A_{p}\right)(\partial e / \partial p)},
$$

$$
\left(\text { being } A_{p}=1-\frac{p}{R} \frac{\partial R}{\partial p} ; A_{T}=1+\frac{T}{R} \frac{\partial R}{\partial T}\right), \quad p=\frac{m R T}{V}
$$

The system of equations (1)-(5), written for each control volume, is solved at each time step by a fourth-order Runge-Kutta scheme. The same technique is employed for a preliminary evaluation of conditions inside intake and exhaust systems.

The system of equations, written for each control volume, is solved at each time step by a fourth-order RungeKutta scheme.

1D Flow Model. As widely described in the quoted papers, this model is based on a one-dimensional unsteady compressible flow through external ducts, therefore, being able to characterize the wave propagation which controls the filling of each cylinder. The mass, momentum, and energy balance equations in conservative form (6) are solved with the TVD method $[9,28]$. The terms $\rho, u, p, E=c_{v} T+u^{2} / 2, H=c_{p} T+$ $u^{2} / 2$ in the equation system (6) are, respectively, density, velocity, pressure, energy, and total enthalpy per mass unit.

Further balance equations for the chemical species and $x_{r}$ and $x_{f}$, which indicate the mass fractions of exhaust gas and fuel, are written to evaluate the gas mixture when the aspiration valve closes. The system is solved by using a "thermodynamic state" table for the simulated gas, this table is included in the software.

The vector of source terms, $\mathbf{S}$, takes into account the effects of the variable section $\Omega$ of the duct $(\alpha=$ $(1 / \Omega)(d \Omega / d t))$, the influence of friction $(f)$, and the heat exchange $(q)$ as described in [6]:

$$
\begin{gathered}
\mathbf{U}_{t}+[\mathbf{F}(\mathbf{U})]_{x}=\mathbf{S}, \\
\mathbf{U}=\left\{\begin{array}{c}
\rho \\
\rho u \\
\rho E \\
\rho x_{r} \\
\rho x_{f}
\end{array}\right\}, \quad \mathbf{F}=\left\{\begin{array}{c}
\rho u \\
\rho u^{2}+p \\
\rho u H \\
\rho u x_{r} \\
\rho u x_{f}
\end{array}\right\}, \\
\mathbf{S}=-\left\{\begin{array}{c}
\rho u \alpha \\
\rho u^{2}(\alpha+2 f / D u /|u|) \\
\rho u H \alpha-4 \frac{q}{D} \\
\rho u x_{r} \alpha \\
\rho u x_{f} \alpha
\end{array}\right\} .
\end{gathered}
$$

Dynamic Reed Valve Model. In order to predict the behavior of the machine also under different operating conditions, the code has to be able to perform a complete simulation of the system based only on geometric parameters. Interalia, a flow model through the reed valves, has been developed, whereby the scheme is quite simple. The valves are placed on the inlet and outlet pipes, where the pressure difference generates a deflection, as a simply supported beam loaded with uniformly distributed load (Figure 2(a)).

Their deflection allows the gas to flow in or out.

For instance, let us consider the outlet valve (Figures 2(a) and 2(b)): the crankcase pressure pushes on one side of the valve, while the discharge pipe pressure acts in the opposite direction. As long as the discharge pipe pressure exceeds the pressure in the crankcase, the reed valve provides 


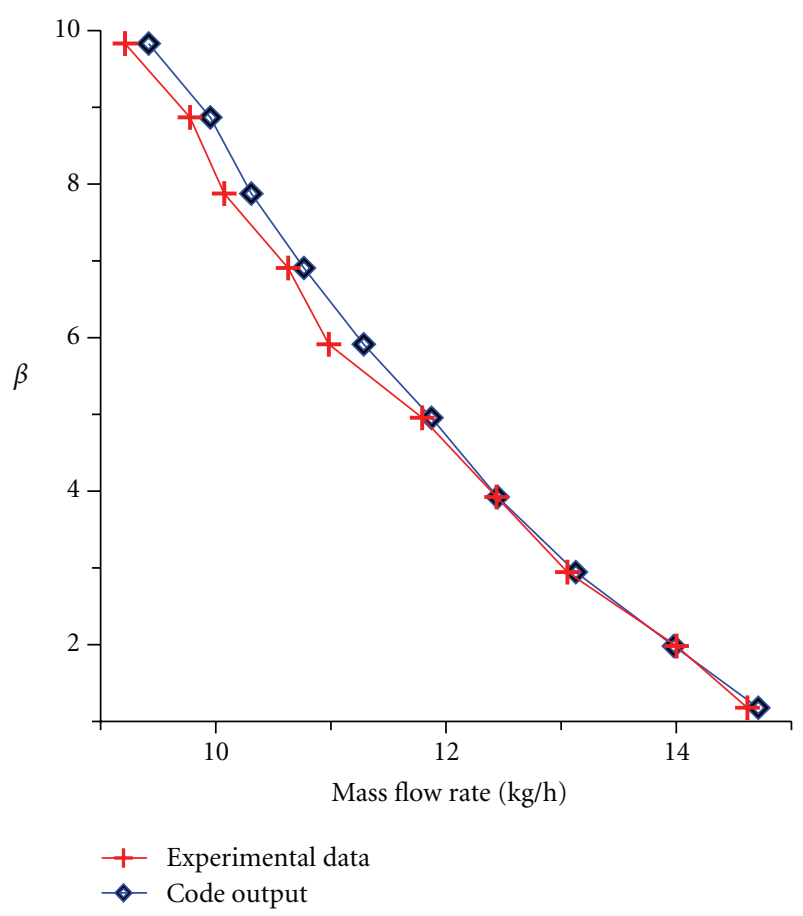

Figure 5: Comparison between numeric and experimental characteristic curves.

the sealing between the two volumes. When the crankcase pressure exceeds that in the discharge pipe, the valve deflects giving a certain cross-sectional flow area.

As mentioned above, the reed valve is seen as simply a supported beam loaded with uniformly distributed load, proportional to the total pressure difference between the crankcase and the pipe and to the width " $d$ " of the reed valve:

$$
q=\left(p_{01}-p_{02}\right) \cdot d
$$

Therefore, the dynamic behavior of the reed valve can be simulated as a mass-spring-damper system, whose differential equation is

$$
m \frac{d^{2} x}{d t^{2}}+\sigma \frac{d x}{d t}+k x=F,
$$

where $m$ is the reed petal mass; $x$ is the instantaneous displacement; $\sigma$ is the damping coefficient; $k$ is the equivalent stiffness; $F$ is the concentrate load which gives the same deflection of the distributed load.

By solving (9) and at the same time the thermodynamic equations of $1 \mathrm{D}$ flow, the deflection is calculated and so the instantaneous cross-section area to estimate the mass flow in the machine.

It is important to specify that, in the case of the considered compressor, the petal deflection is limited by two steel brackets which allow a maximum lift of $2 \mathrm{~mm}$. The equivalent stiffness $k$ can be estimated by imposing the same deflection between the two schemes in (Figure 3), being $X_{D}$ the deflection caused by a concentrated load $F=k x$.

At last, the result is $k=48\left(E I / l^{3}\right)$. In the absence of experimental data, a constant flow coefficient has been

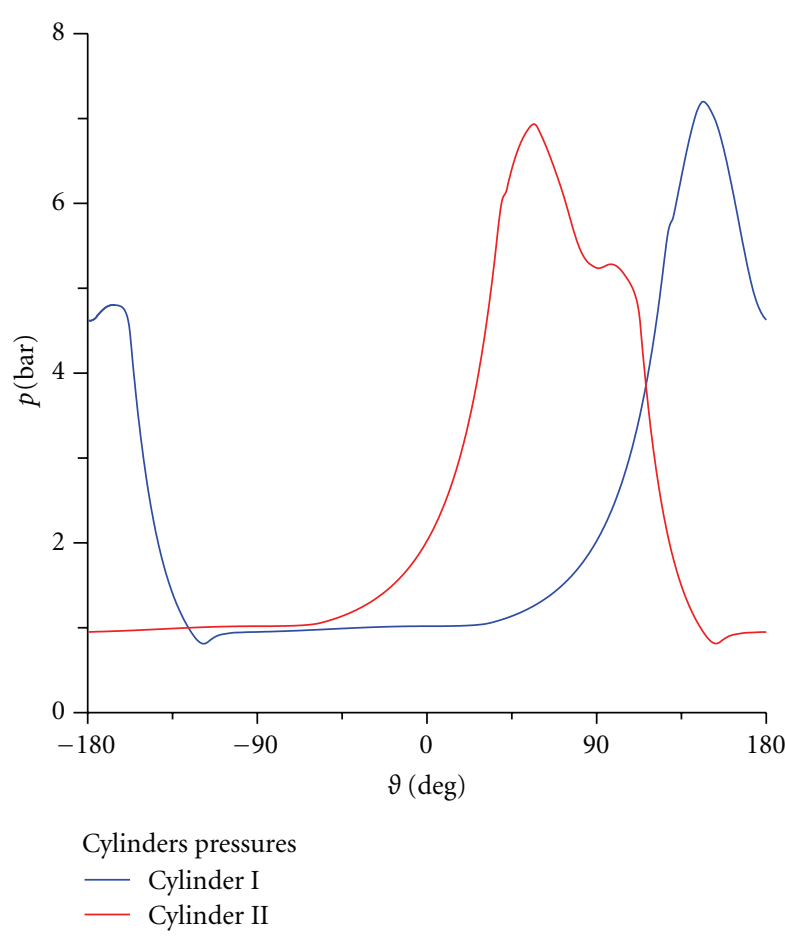

Figure 6: Pressure in the cylinders at $\beta=5$.

used in all load conditions. The flux is considered isentropic until the restricted section, then isobaric downstream of the valve. The pressure in the throat is obtained by the mass flow balance between the cylinder and the adjacent pipe. A thorough validation of such a submodel is difficult to perform due to experimental complications, however, an indirect validation has been made by confronting the actual mass flow rate displaced by the compressor and the one determined by the code. The satisfying correspondence reached justifies the use of this submodel.

Boundary Conditions. The numerical simulation of the flow in a reciprocating engine duct is characterized by timevarying boundary conditions, in terms of both valve flow areas and thermodynamic properties inside the neighboring devices. In addition, a proper flow calculation at the end stations ensures a correct evaluation of the in-cylinder conditions during the gas exchange phases. The solution of end boundaries is performed by solving the typical compatibility equations along mach and path lines for a flow entering or exiting the duct.

A classical quasisteady approximation was assumed as to ensure the consistency with the flow through the intake and exhaust valves, which take place under either subsonic or sonic flow conditions.

Input of the Code and Solutions. To perform a simulation, the code requires in input a series of volumes, which represent the machine setup, their geometries, and initial conditions: each element is connected to other elements or to the external environment through links which can be constant 

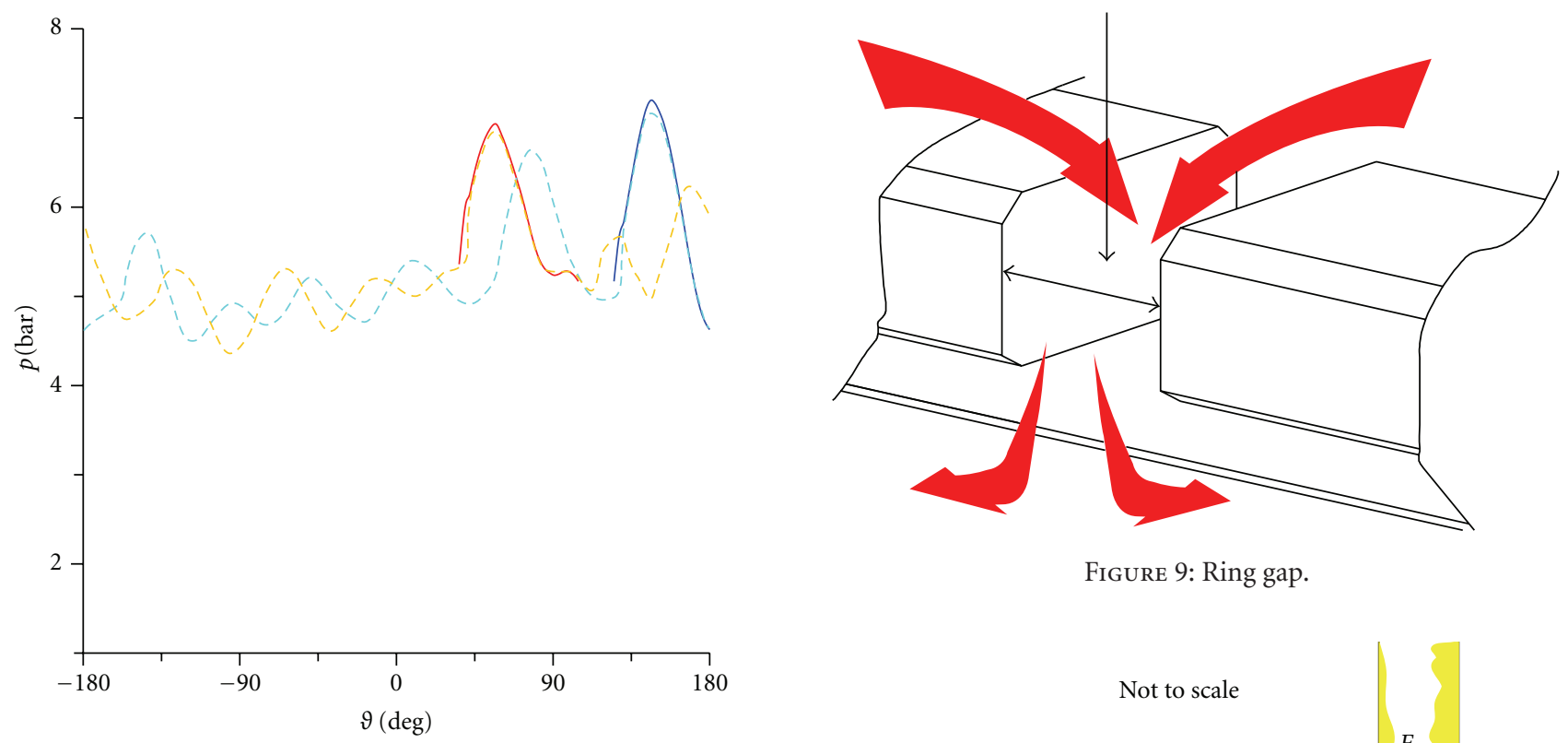

FIgURE 9: Ring gap.

Discharge pressure
- Cyl. I during discharge
- - Discharge pipe cyl. I
— Cyl. II during discharge
Discharge pipe cyl. II

Figure 7: Pressure in the discharge pipes at $\beta=5$.

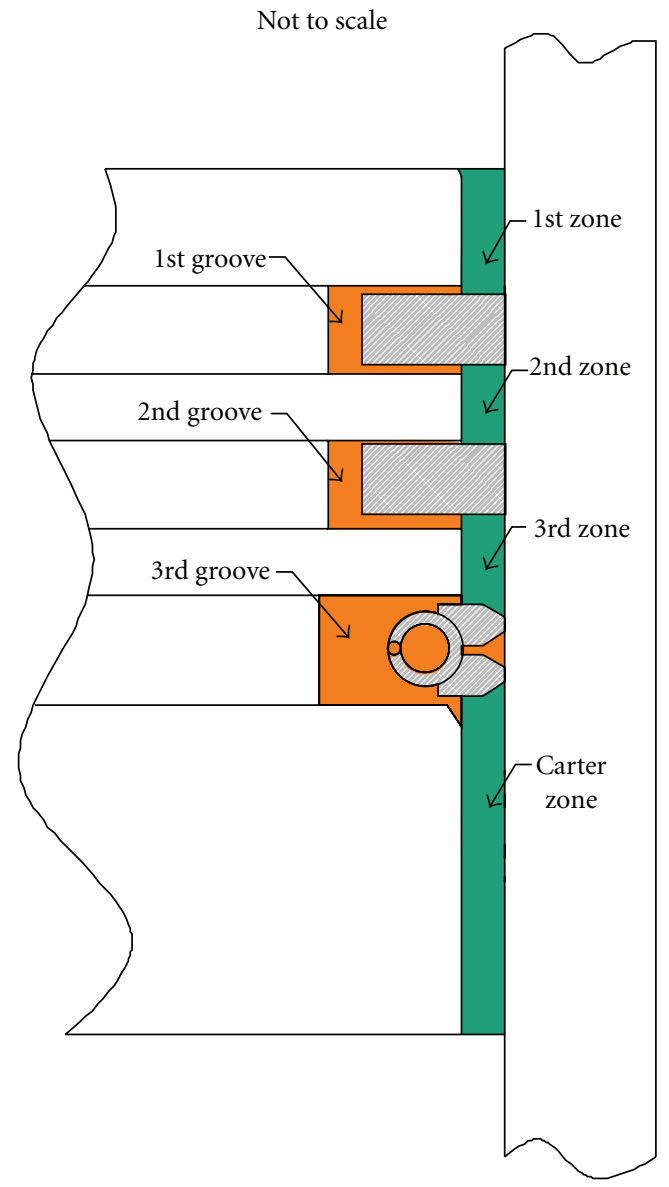

FIgURe 8: PRL system subdivision.

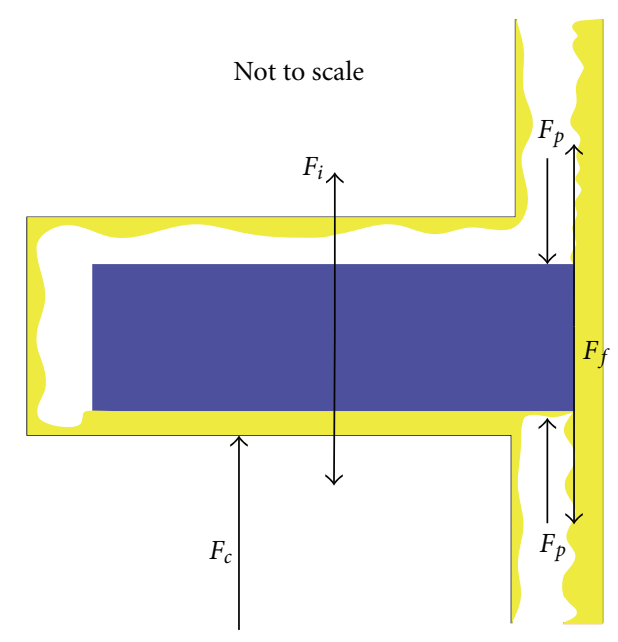

Figure 10: Axial forces on the segment.

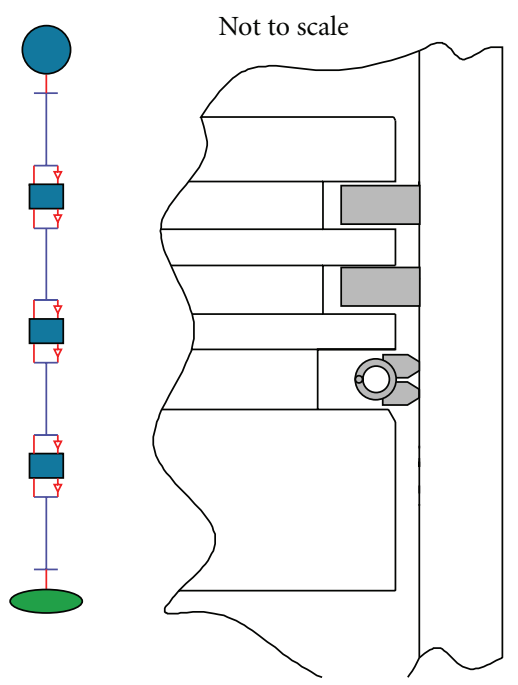
External volume
$\longmapsto 1$ 1D element
OD variable volume
$\rightarrow$ Valve link
0D constant volume
—Constant link

FIGURE 11: 0D-1D PRL scheme. 


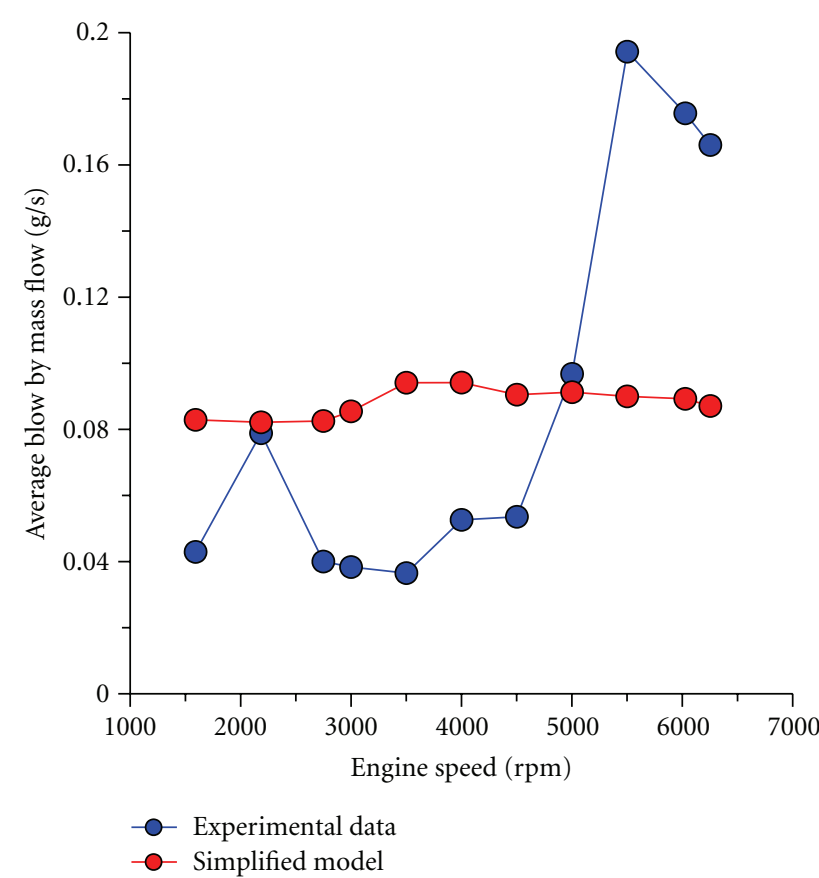

FIGURE 12: Experimental-simplified model Blow-by comparison.

cross section, ports, or valves (automatic or controlled). The boundary volumes are linked to sets which are at fixed thermodynamic conditions (e.g., ambient conditions). As results, the code provides, in arrays, both instantaneous and medium values of the thermodynamic parameters in each volume.

\subsection{D-1D Compressor Scheme and Comparison with Exper-} imental Data. Once the test-bed layout and the ducts geometries are known, it is possible to create a $0 \mathrm{D}-1 \mathrm{D}$ scheme (Figure 4) in input to the code: the inlet pipes are connected to the external environment set AA, while the duct SS is connected to a set in the same conditions of the holding tank TNK. On the discharge line, R1 and E2 converge in R2 bringing together the flows coming from the two cylinders.

A thermofluid-dynamic simulation of the 10 stationary tests in Table 1 was performed by the code, and in Figure 5 it is shown as a comparison between the numeric characteristic curve (blue line) and the experimental one (red line). The $x$ axis is associated to the mass flow rate (both measured and predicted) in the SR volume, the $y$-axis to the compression ratio $\left(\beta=p_{\mathrm{TNK}} / p_{\mathrm{AA}}\right)$.

The characteristic is predicted pretty well, the curves (Figure 5) seem to overlap with a slight divergence for $\beta>5$, this problem is possibly due to the leaks of the valves and the piston rings in the real case.

In Figure 6, displayed are the predicted pressure diagrams in the two cylinders, and it shows that in the discharge phase of the cycle, pressure is highly variable because of the pressure waves that propagate along the discharge line as observed from the pressure curves of the discharge pipes (dashed lines, Figure 7).

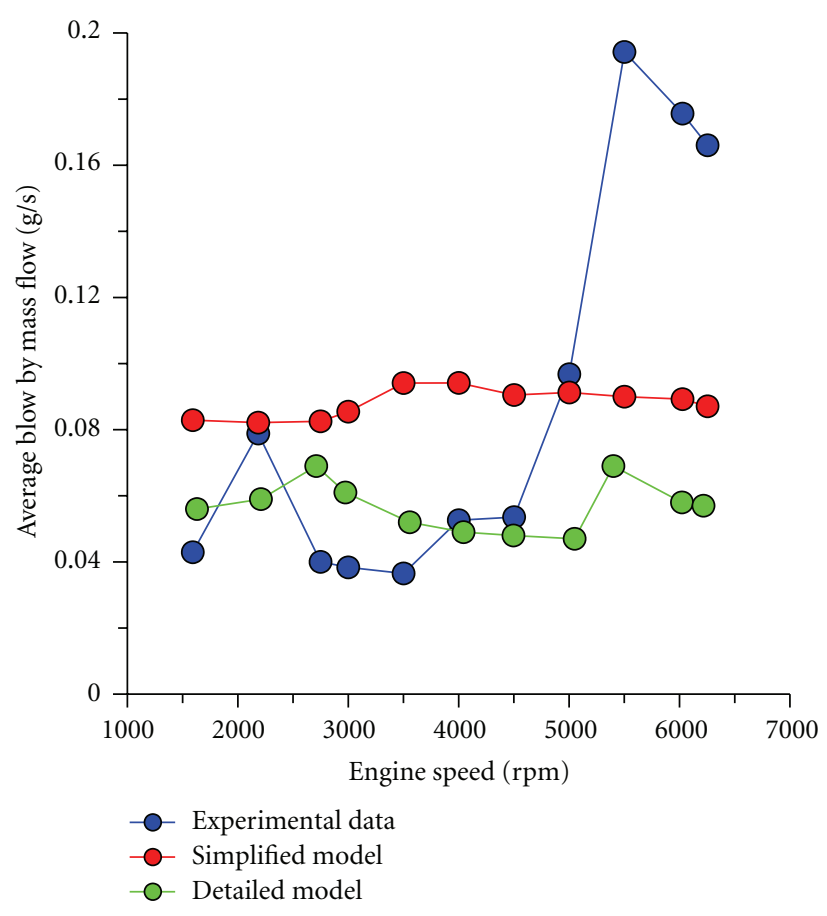

FIgURE 13: Blow-by models comparison.

As already mentioned, the compressor is a twin V-unit, and this means that the discharges of the cylinders take place with an interval of $90^{\circ}$ (Figure 6).

It is clear that this phenomenon forces the compressor to reach pressures way higher (about 7 bar cycle II, about 7.2 bar cycle I) of the medium discharge pressure (about 5.3 bar in $\mathrm{S} 1$ and S2 ducts).

The liaison between the two cycles is clear, observing in Figure 7 how the discharge phase of cylinder II generates a pressure wave (peak at about $80^{\circ}$ of light blue-dashed line) that affects the pressure during the discharge phase in cylinder I. This occurs due to the discharge happening in a volume at highly variable pressure. The same effects are more attenuated for cylinder II as there is more time since the closure of cylinder I discharge, which allows the pressure waves to dump.

This analysis shows how, by this modeling approach, it is possible to properly design the pipes upstream and downstream the compressor in order to reduce the pressure waves that, beside requiring more power from the electric engine, decrease the mass flow rate, and cause an increase in the noise during operation.

\section{Blow-By}

In order to improve the accuracy of the modeling approach, it was considered to weigh the gas leakage due to an imperfect sealing which took place between the piston and cylinder, this phenomenon in literature is termed "blow-by" [26].

The piston sealing system consists essentially of segments, also known as piston rings; these are open metal flattened rings, and they have two main functions: to provide 


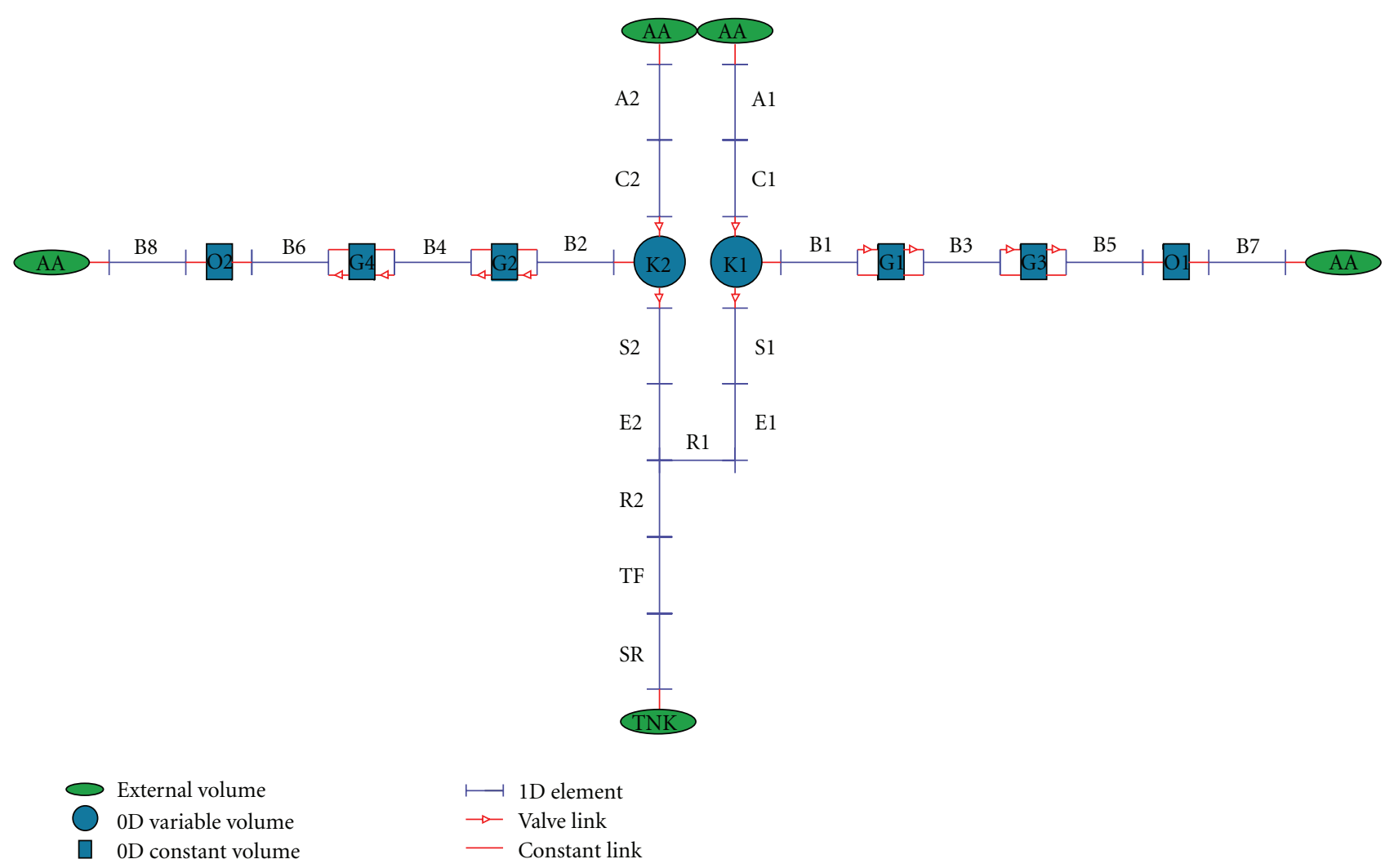

FIGURE 14: Final 0D-1D scheme for the compressor with blow-by volumes.

a certain tightness in the compression chamber, minimizing energy losses to regulate and level the lubricant distribution on the cylinder liner, minimizing both consumption and friction losses. The Piston-Ring-Liner system (PRL) can be split into different volumes identified by their relative position beside the sealing segments: the first zone, near the head of the piston; the second and third zones, located under the two rings; the crankcase zone, placed between the OCR and the crankcase; the first, second grooves and the OCR groove, which are the volumes placed behind the segments (Figure 8).

To report this subdivision within a scheme that can be interpreted by the code used, equivalent volumes and capacities are identified and they have been implemented within the machine framework. As already mentioned, two kinds of volume can be identified in the PRL system:

(i) the "zones" that are the annular pipes identified by the clearance between the piston diameter and the cylinder shell,

(ii) the "grooves" that are the volumes identified by the distance between the inner surface of a ring and the bottom of the piston groove.

Please note that in this work secondary movements of the piston are not taken into account for the identification of the flow sections, which cause a section variance moment by moment during a cycle. Due to the "zones" having an annular section, it was identified in having an equivalent diameter and a corrective coefficient of the friction factor $f$, so it was possible to match the pressure drops in the pipe with the real pressure drop [29].

If the section is not circular, the equivalent hydraulic diameter has to be used; this new diameter is obtained by the equation:

$$
D_{\mathrm{eq}}=\frac{4 \cdot A}{P} \text {. }
$$

So, for annular section pipes [29]:

$$
D_{\mathrm{eq}}=D_{e}-D_{i}
$$

For annular section pipes, the correction coefficient of the friction factor may be considered [29],

$$
c_{f}=\frac{f_{\text {annular }}}{f_{\text {circular }}}=1.05 .
$$

Regarding the "ring gap," there are very complex phenomena taking place, as flows from two zones and from the inside of the groove cross themselves, as well as the relative shift of the segment that can cause an accumulation of lubricant in the hole.

Since the purpose of this work is not the particular analysis of the flow through the gap, a simplified model was chosen: in Figure 9, illustrated is the gap geometry and the gas flow between one zone to another through the interspaces. 


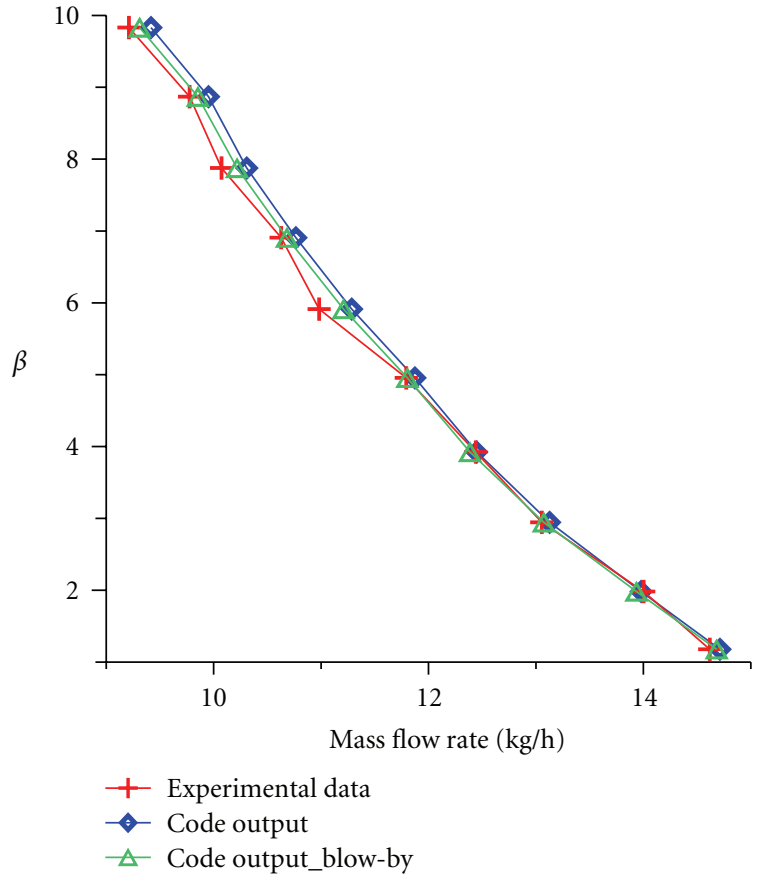

FIGURE 15: Characteristic curves comparison.

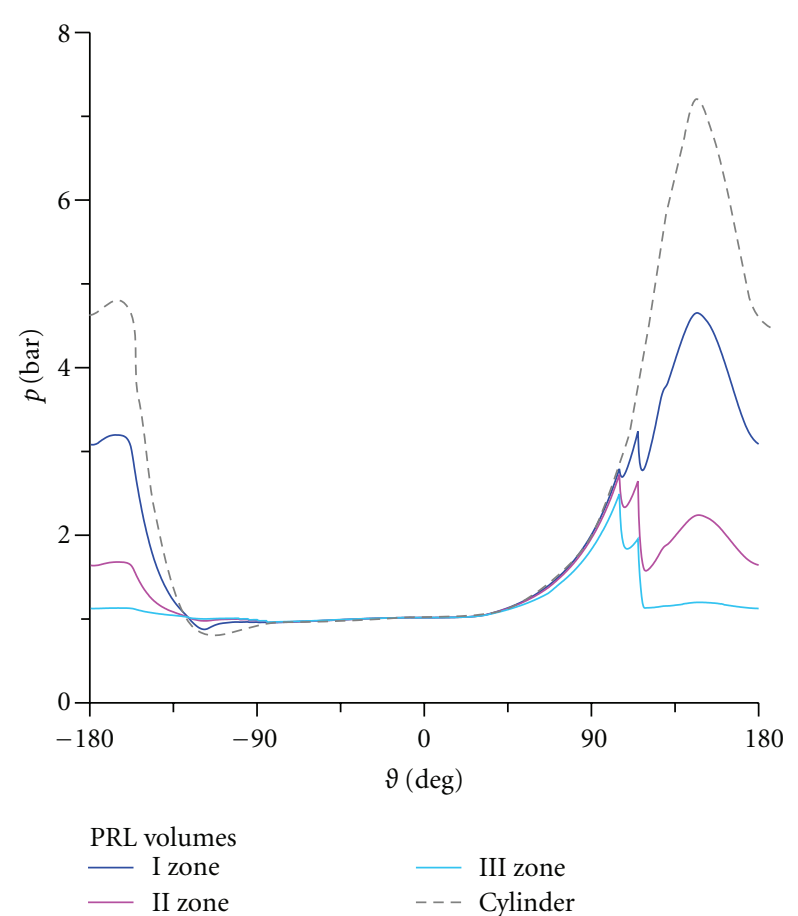

Figure 16: Pressure analysis in PRL volumes, $\beta=5$, stable model.

The pressure inside the gap is assumed equal to the pressure inside the groove, while the gas flow that passes through the gap can be expressed by the isentropic flow equations through an orifice, and so it was decided to include this element in the geometric scheme as a port between the groove and the linked zones [30]. The flow through the gap sets the pressure difference which is established between different zones, and therefore, also affects the ring dynamic.

4.1. Ring Dynamics inside the Grooves. The gas flow is closely related to the rings dynamic, it works like a port that opens only when it is separated from both walls of the groove. It is a prohibitive work predicting the opening without any experimental feedback, just thinking that three components of a segment with relative displacement can be identified: axial, radial, and angular. Each one is caused by a system of forces which vary moment by moment. This paper, with the limits of a one-dimensional model, has been concentrated on the axial displacement. Axial forces acting on a segment during a cycle are identified as follows (Figure 10):

(i) $F_{p}$ : gas and oil pressure above and below the segment;

(ii) $F_{f}$ : friction between cylinder liner and piston;

(iii) $F_{i}$ : inertia due to the piston alternative motion;

(iv) $F_{c}$ : contact force between segment side and groove.

These forces resultant cause the relative axial displacement of the segment inside the groove [31].

Starting from the wider literature about "blow-by" in internal combustion engines, to find an analogy in the rings dynamic, it is possible to hypothesize the two displacement models for the rings:

(i) Stable Model: all segments displacement follows the oscillating masses inertia force trend;

(ii) Flutter Model: forces resultant during compression final phase change sign several times, causing irregular oscillations of the segment inside the groove.

These two models are used to hypothesize and impose the shift law of the "valve ports" described in the subsequent paragraph.

4.2. PRL System Summery and $0 D-1 D$ Schemes. At this point, a synthesis of the PRL system was reached, consisting of an ensemble of zero and one-dimensional volumes. Zones, according to the assumptions made previously, are annular section pipes, to represent as good as possible the grooves it was chosen, instead, a zero-dimensional capacity, linked to the adjacent volumes through two different kinds of section:

(i) a variable section due to the segment axial displacement, which as said before, can be represented by some "controlled valve" port type that has to be set with the more appropriated shift law;

(ii) a constant section port which represents not only the flow through the ring gap, but also the poor seal between shell and segments, due to an uneven surface.

In addition, to take into account dissipative phenomena that happens in the flow through the gap, a fixed flow factor is imposed to the constant section link. 


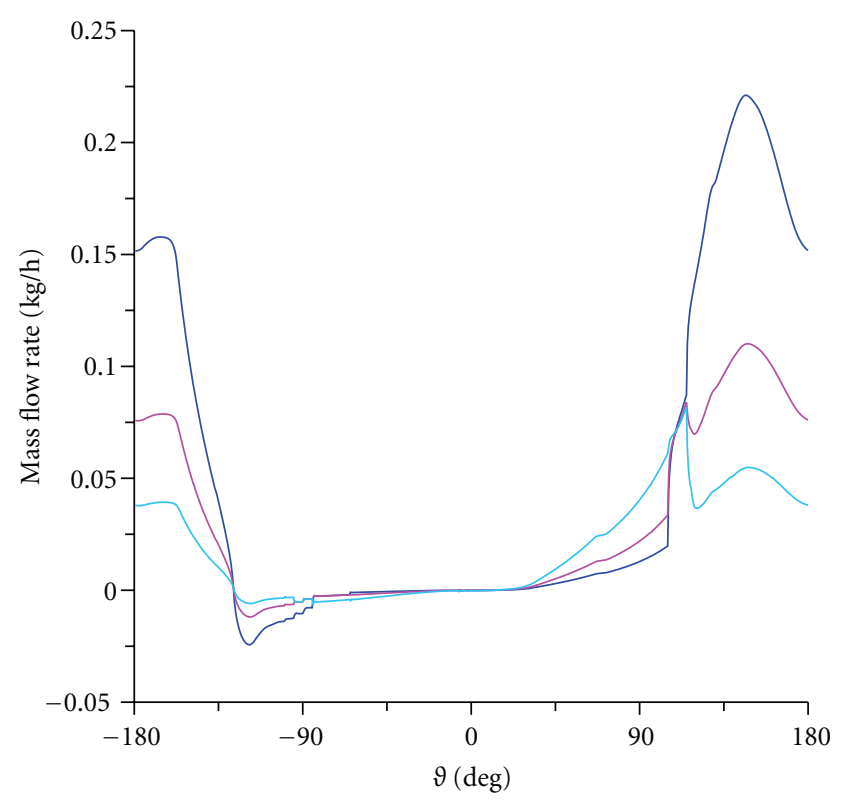

PRL volumes

- I zone

- II zone

_ III zone

FIGURE 17: blow-by flow rate in PRL volumes, $\beta=5$, stable model.

TABLE 2: Features of the analyzed engine.

\begin{tabular}{lc}
\hline Engine type & Twin 4T \\
\hline Cylinder number & 2 \\
\hline Displacement & $908 \mathrm{~cm}^{3}$ \\
\hline Stroke/Bore/Conn. Rod length & $(86 / 82 / 133.55) \mathrm{mm}$ \\
\hline Compression ratio & 11 \\
\hline
\end{tabular}

4.3. PRL Scheme Validation. To validate the PRL scheme system of the "blow-by" just described, several schemes have been tested for a 4-stroke two-cylinder engine (Table 2) on which experimental data was reported in relation to the "blow-by" flow rate. First of all, the PRL circuit of "blowby" was schemed by a single $1 \mathrm{D}$ pipe with a circular section, its diameter was considered to be the average of the quadratic diameters to receive the experimental flow rate of "blow-by." The comparison between the experimental flow rate and the data taken thanks to the simplified scheme characterized by a single $1 \mathrm{D}$ pipe and constant diameter equal to the medium quadratic is shown in Figure 12.

From the diagram in Figure 12, it is possible to notice how the model gives, obviously, an average value of the "blow-by" flow rate coherent enough with experimental data, but not so indicative for each single loading rate.

This is proof that the rings dynamic role is essential to predict the "blow-by" flow rate. So, it was realized a detailed PRL system scheme according to the logic described for the compressor in the previous paragraph and illustrated in Figure 11. This scheme has been realized by having data of the geometry of the piston, cylinder, piston rings, and the hot coupling between cylinder and piston.

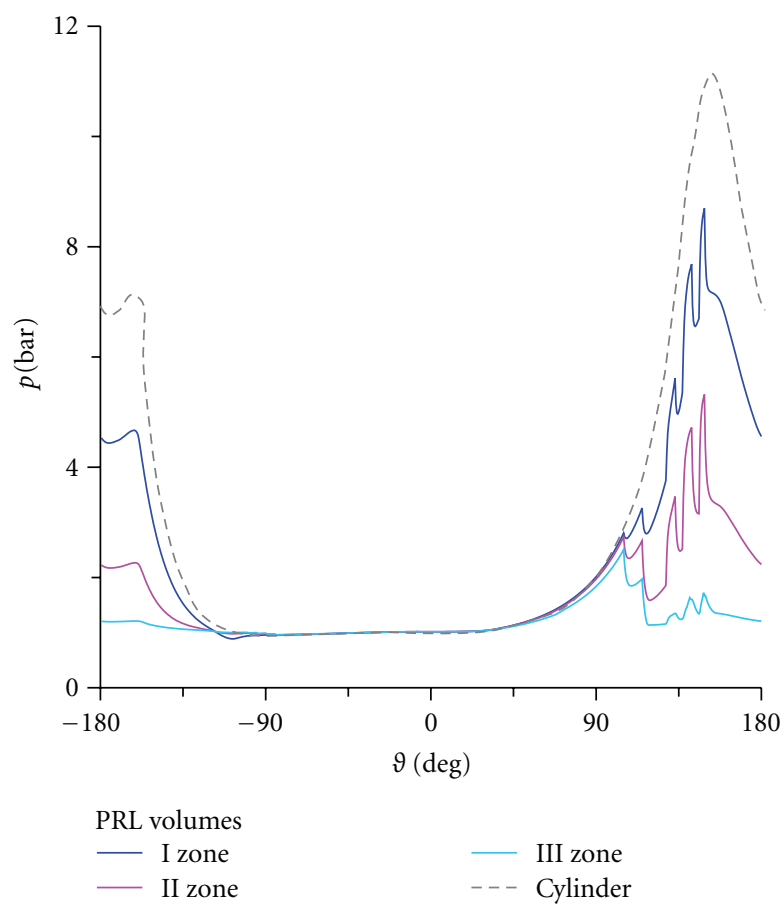

FIgURE 18: Pressure in PRL volumes, $\beta=8$, flutter model.

The rings motion in their own grooves was imposed, according to the stable model, by two symmetric shift laws interpolated on 720 degrees of the crank angle, based on the above-mentioned stable model: the piston rings motion have been imposed according to experimental measurement reported in [26].

The blow-by mass flow rate obtained thanks to the PRL scheme in relation of rpm is shown in Figure 13 (red line); although it seems that the detailed scheme does not offer better results than the simplified one, note that the input of the first is only the PRL geometry, while the simplified scheme needs experimental data.

By comparing (in Figure 13) the (red line) with the experimental data (blue line), it can be noted that the flow rate is well predicted till $4500 \mathrm{rpm}$; over this rpm probably the flutter phenomenon intervenes [26], which should influence seriously the "blow-by" flow rate. It is in the authors intention to realize in the next work a piston rings dynamic model, so it can be verified if the flow rate rise over $4500 \mathrm{rpm}$ is due to flutter phenomenon.

\section{Blow-By Model for a Compressor}

After the analysis conducted on the alternative internal combustion engine, the detailed PRL scheme (Figure 11) with imposed piston rings dynamic, adherent to the stable model, was considered sufficiently predictive to conduct some analysis on the "blow-by" phenomenon effects on the compressor characteristic curve. Here, in Figure 14 is shown the complete $0 \mathrm{D}-1 \mathrm{D}$ scheme used by the code to simulate the normal compressor displacement (to the TNK set) and the leakage through the ring pack system (to lateral AA sets). 


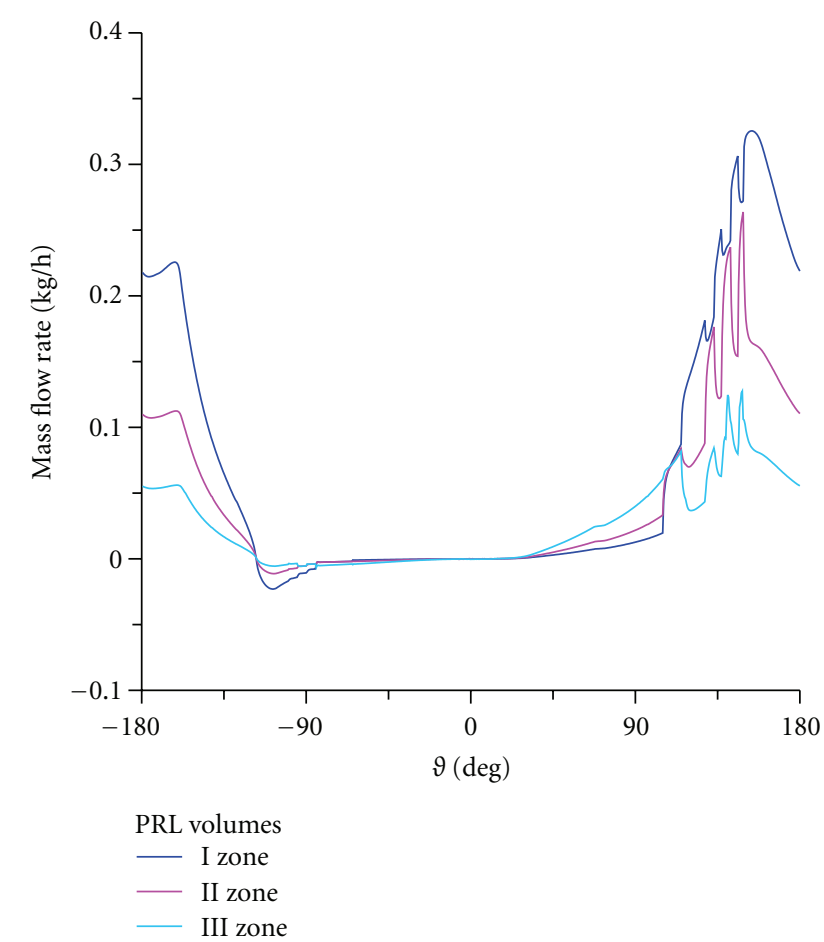

FIgURE 19: Mass flow rate in PRL volumes, $\beta=8$, flutter model.

In Figure 15, the obtained characteristic considering the effect of the "blow-by" in the compressor (light green line) is compared with the characteristic previously obtained without introducing the "blow-by" scheme. The results show that the curves are practically coincident till $\beta=5$, which means that the phenomenon is practically irrelevant when the compressor works with low compression ratios. For higher values, instead, the characteristic obtained simulating the "blow-by" phenomenon in the compressor maintains a slope nearly coincidental with the experimental curve, better explaining the real machine behavior.

In Figure 16, the pressure trends in the cylinder and in the zones of the PRL system, called "grooves" that is in the piston rings accommodation, are reported. In Figure 11, the instant pressure in these zones follows the inside cylinder pressure trend, reaching, however, peaks necessarily smaller.

As mentioned previously, this is due to a strongly influenced flow rates as a result of the ring gap, which in spite of the strong losses, realizes a continuous link between the compression chamber and the zones between piston and cylinder. As we move from the cylinder to the crankcase, the pressure curves tend to assume the value of ambient pressure, which is the condition (assumed uniform) in the crankcase. In coincidence of the segments displacement (approx. 112 degree, in Figure 16), there is a pressure drop due to the opening of superior and inferior channels of each groove, generating a flow rate peak (Figure 17). Compression occurs during the opening of the passage through the piston rings, with a displacement toward the segment upper edge.

In Figure 17, it is also observed that there are some cycle phases where there is a reverse "blow-by" (about 135 degree).
In particular, at the end of the expansion phase, when the first zone is at a pressure higher than the compression chamber, part of the gas reflows till the pressure difference vanishes. A similar argument is also valid for the intermediate zones, which along whole the intake phase are affected by a reversal of the "blow-by" flow even if it is minimum.

From Figure 16, it also observed that there are large intervals where, between the cylinder and the various zones and between themselves, wide $\Delta p$ are generated, which could generate forces opposite to inertia force on the segment, leading to the flutter phenomenon.

Now, it is possible to perform an interesting analysis of the parameters in PRL volumes in case of ring flutter. A series of fluctuations has been hypothesized for the first ring during the pressure raise in the case of $\beta=8$. In Figure 18, where the fluctuations take place, a pressure drop can be observed, especially in the first two zones.

As can be seen in Figure 19, the blow-by mass flow rate has no more this peak in one single point, but there are several peaks which take place in the exact same time that the ring flutters. This phenomenon shows that the blow-by is highly influenced by the ring dynamics, and in particular way if the ring is not stable during the cicle.

\section{Conclusions}

This work has been developed to improve the accuracy of the simulation of the CFD phenomenon in the volumetric machines. A $1 \mathrm{D}$ procedure is proposed and validated with the experimental data taken from a volumetric alternative compressor. In addition, a PRL system scheme was implemented for the "blow-by" simulation. This scheme, based on the "stable" dynamic imposed to the segments, has enabled an improvement in the characteristic reproduction of the compressor, besides; it was possible to carry out a first CFD analysis inside the cavity between the cylinder and piston. In light of the encouraging results obtained, it is considered appropriate that a development of a predictive dynamic model of the segment displacement to be implemented on the 0D-1D simulation software, so it will improve the model prediction in all operation conditions.

\section{Acronyms}

0D: Zero-dimensional

1D: One-dimensional

TVD: Total variation diminishing

PRL: Volumes between piston ring and liner

OCR: Oil control ring.

\section{Nomenclature}

\section{Latin}

$p_{\mathrm{amb}}$ : Ambient pressure

$T_{\mathrm{amb}}$ : Ambient temperature

$p_{\text {tnk: }}$ : Tank pressure

$T_{\text {oil }}$ : Oil temperature

$p_{01}$ : In-cylinder pressure on reed valve 
$p_{02}$ : $\quad$ Outlet pressure on reed valve

$p_{\text {TNK }}$ : Tank pressure

$p_{\text {AA }}$ : Ambient pressure

$V_{3}$ : $\quad$ Cylinder top clearance

$V: \quad$ Displacement volume

$D_{\text {eq }}$ : Equivalent hydraulic diameter

$f_{\text {annular }}$ : Annular cross-section friction factor

$f_{\text {circular }}$ : Circular cross-section fiction factor.

Greek

$\beta: \quad$ Compression ratio

$\beta_{\text {MAX }}$ : Theoretical maximum compression ratio.

\section{References}

[1] S. A. Tassou and T. Q. Qureshi, "Comparative performance evaluation of positive displacement compressors in variablespeed refrigeration applications," International Journal of Refrigeration, vol. 21, no. 1, pp. 29-41, 1998.

[2] E. Winandy, C. Saavedra O, and J. Lebrun, "Simplified modelling of an open-type reciprocating compressor," International Journal of Thermal Sciences, vol. 41, no. 2, pp. 183192, 2002.

[3] C. O. R. Negrão, R. H. Erthal, D. E. V. Andrade, and L. W. D. Silva, "A semi-empirical model for the unsteadystate simulation of reciprocating compressors for household refrigeration applications," Applied Thermal Engineering, vol. 31, no. 6-7, pp. 1114-1124, 2011.

[4] C. D. Pérez-Segarra, J. Rigola, and A. Oliva, "Modeling and numerical simulation of the thermal and fluid dynamic behavior of hermetic reciprocating compressors-part 1: theoretical basis," HVAC and R Research, vol. 9, no. 2, pp. 215235, 2003.

[5] C. D. Pérez-Segarraa, J. Rigolaa, and A. Olivaa, "Modeling and numerical simulation of the thermal and fluid dynamic behavior of hermetic reciprocating compressors-part 2: experimental investigation," HVAC\&R Research, vol. 9, no. 2, pp. 237-249, 2003.

[6] F. Bozza, M. Manna, and A. Pascarelli, "Un approccio misto 0D/1-D per la simulazione di M.C.I. a 2 tempi," in Atti del $49 \circ$ Congresso Nazionale ATI, vol. 2, pp. 1835-1847, SGE-Padova, Settembre 1994.

[7] M. N. Srinivas and C. Padmanabhan, "Computationally efficient model for refrigeration compressor gas dynamics," International Journal of Refrigeration, vol. 25, no. 8, pp. 10831092, 2002.

[8] D. Woollatt, "Factors affecting reciprocating compressor performance," Hydrocarbon Processing, vol. 72, no. 6, pp. 657-65, 1993.

[9] M. Manna, A three dimensional high resolution compressible flow solver, Ph.D. thesis, Catholic University of Leuven-Von Karman Institute for Fluid Dynamics, 1992, also in Technical Note 180, VKI, 1992.

[10] F. Bozza, R. Tuccillo, and D. deFalco, "A two-stroke engine model based on advanced simulation of fundamental processes," in Proceedings of the International Off-Highway \& Powerplant Congress \& Exposition, SAE Paper no. 952139, Milwaukee, Wis, USA.

[11] Society of Automotive Engineers, Design and Emissions of Small Two- and Four-Stroke Engines, SAE SP-1112, Society of Automotive Engineers, 1995.
[12] F. Bozza, M. C. Cameretti, and R. Tuccillo, "Numerical simulation of in-cylinder processes and duct flow in a lightduty diesel engine," in Proceedings of the 4th International Symposium on Small Diesel Engines, pp. 24-25, Varsavia, Poland, pubblicato su Journal of Polish Cimac, vol. 2, no. 1, pp. 51-66, 1996.

[13] F. Bozza, A. Senatore, R. Tuccillo, and A. Gimelli, "Caratterizzazione sperimentale e teorica del funzionamento di un motore diesel di concezione avanzata," Rivista-ATAAssociazione Tecnica dell'Automobile, pp. 117-129, 1997.

[14] F. Bozza, M. C. Cameretti, A. Senatore, and R. Tuccillo, "Experimental investigation and numerical modelling of an advanced turbocharged D.I. diesel engine," in Proceedings of the SAE International Congress \& Exhibition, SAE Paper no. 970057, pp. 19-31, February 1997, pubblicato anche sul volume "Engine Modeling", SAE SP-1255, 1997.

[15] F. Bozza, M. C. Cameretti, A. Senatore, and R. Tuccillo, "A flow and a combustion model for NOx emission evaluation in a turbocharged diesel engine," in Proceedings of the $3 \mathrm{rd}$ International Conference on Internal Combustion Engines: Experiments and Modeling (ICE '97), pp. 153-162, Capri, Italy, Settembre 1997.

[16] F. Bozza, M. C. Cameretti, and R. Tuccillo, "Numerical simulation of transient behaviour of an EGR equipped turbocharged engine," in Proceedings of the 4th International Conference on Internal Combustion Engines: Experiments and Modeling (ICE '99), pp. 313-320, Capri, Italy, Settembre 1999.

[17] F. Bozza, A. Senatore, and R. Tuccillo, "Integrated numericalexperimental method for the analysis of in-cylinder processes in an EGR equipped diesel engine," in Proceedings of the Fall Technical Conference of the ASME Internal Combustion Engine Division, pp. 16-20, Ann Arbor, Mich, USA, Ottobre 1999.

[18] F. Bozza, A. Gimelli, and R. Tuccillo, "The control of a VVAequipped SI engine operation by means of $1 \mathrm{D}$ simulation and mathematical optimization," in Proceedings of the SAE International Congress \& Exhibition, vol. SP-1692 of SAE Paper no. 2002-01-1107, Detroit, Mich, USA, 2002, Published also in SAE Transactions, Journal of Engines, vol. 111, pp. 1790 -1801, 2003.

[19] F. Bozza and A. Gimelli, "A comprehensive 1D model for the simulation of a small-size two-stroke SI engine," in Proceedings of the SAE World Congress \& Exhibition on Modeling of SI and Diesel Engines, vol. SP-1830 of SAE Paper no. 2004-01-0999, pp. 165-177, March 2004, Published also in the on the $S A E$ Transactions, Journal of Engines, section 3, July 2005.

[20] F. Bozza, A. Gimelli, V. Pianese, S. Martino, and R. Curion, "An acoustic design procedure for intake systems: 1D analysis and experimental validation," in Proceedings of the SAE World Congress \& Exhibition on Modeling of SI and Diesel Engines, vol. SP-1867, pp. 139-147, March 2004, Published also in Transactions Journal of Passenger Cars, vol .6, pp. 172-180, 2005.

[21] D. Siano, F. E. Corcione, F. Bozza, A. Gimelli, and S. Manelli, "Characterization of the noise emitted by a single cylinder diesel engine: experimental activities and 1D simulation," in Proceedings of the SAE Noise and Vibration Conference and Exhibition, SAE Paper no. 2005-01-2483, Grand Traverse, Mich, USA, May 2005.

[22] F. Bozza, A. Gimelli, R. Piazzesi, F. Fortunato, V. Pianese, and D. Siano, "The prediction of the performance and gasdynamic noise emitted by a medium-size spark-ignition engine by means of 1D and 3D analyses," in Proceedings of the SAE World Congress \& Exhibition on Modeling of SI and Diesel Engines, vol. 
SP-2079 of SAE Paper no. 2007-01-0380, Detroit, Mich, USA, April 2007.

[23] F. Bozza, A. Gimelli, L. Strazzullo, E. Torella, and C. Cascone, "Steady-state and transient operation simulation of a, "downsized" turbocharged SI engine," in Proceedings of the SAE World Congress \& Exhibition on Modeling of SI and Diesel Engines, vol. SP-2079 of SAE Paper no. 2007-01-0381, Detroit, Mich, USA, April 2007.

[24] F. Bozza, A. Gimelli, L. Andreassi, V. Rocco, and R. Scarcelli, "1D-3D analysis of the scavenging and combustion process in a gasoline and natural-gas fuelled two-stroke engine," in Proceedings of the SAE World Congress \& Exhibition on Modeling of SI and Diesel Engines, SAE Paper no. 2008-011087, p. 303, Detroit, Mich, USA, April 2008, On SP-2156 (Modeling of SI and Diesel Engines, 2008), Journal SAE Technical Paper, pp. 303-316.

[25] F. Bozza and A. Gimelli, "Unsteady 1D simulation of a turbocharger compressor," in Proceedings of the SAE World Congress \& Exhibition on Modeling of SI and Diesel Engines, SAE Paper no. 2009-01- 0308, pp. 67-76, Detroit, Mich, USA, April 2009, Also in SAE International Journal of Engines, vol. 2, pp. 189-198, October 2009.

[26] T. Tian, Modeling the performance of the piston ring-pack in internal combustion engines, Ph.D. thesis, Massachusetts Institute of Technology, 1997.

[27] O. Pennacchia, Studio di compressori alternativi dotati di manovellismo non convenzionale, Tesi di laurea in Ingegneria Meccanica, Università di Napoli Federico II, 2003.

[28] A. Harten, "On a class of high resolution total variation stable finite difference schemes," Journal of Computational Physics, vol. 21, pp. 21-23, 1984.

[29] D. S. Miller, Internal Flow Systems, 1990.

[30] L. Liu, Modeling the performance of the piston ring pack with consideration of non-axisymmetric characteristics of the power cylinder system in IC Engines, Ph.D. thesis, Massachusetts Institute of Technology, 2005.

[31] K. Jia, A coupled model for ring dynamics, gas flow and oil flow through the ring grooves in IC engines, Ph.D. thesis, Massachusetts Institute of Technology, 2009. 

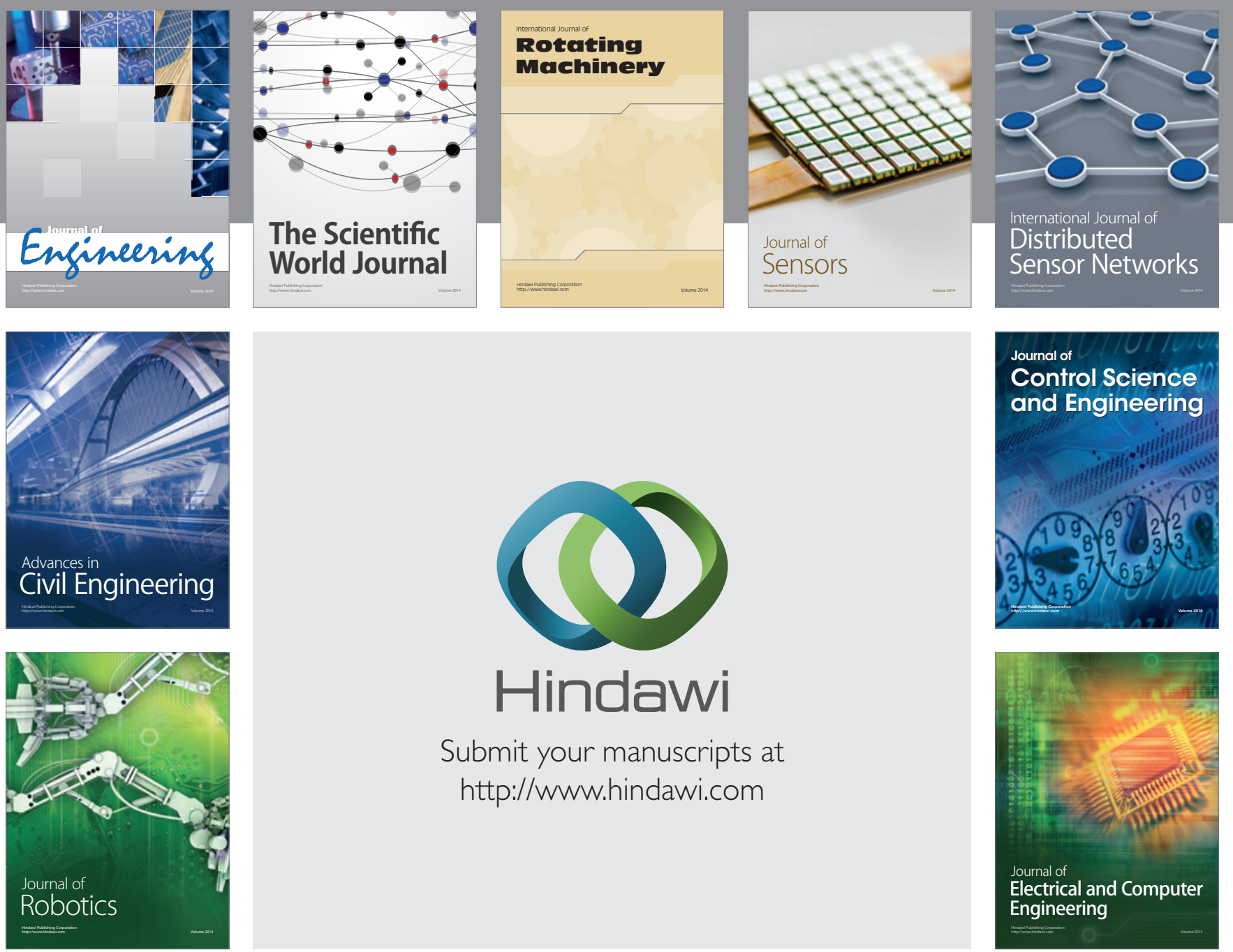

Submit your manuscripts at

http://www.hindawi.com
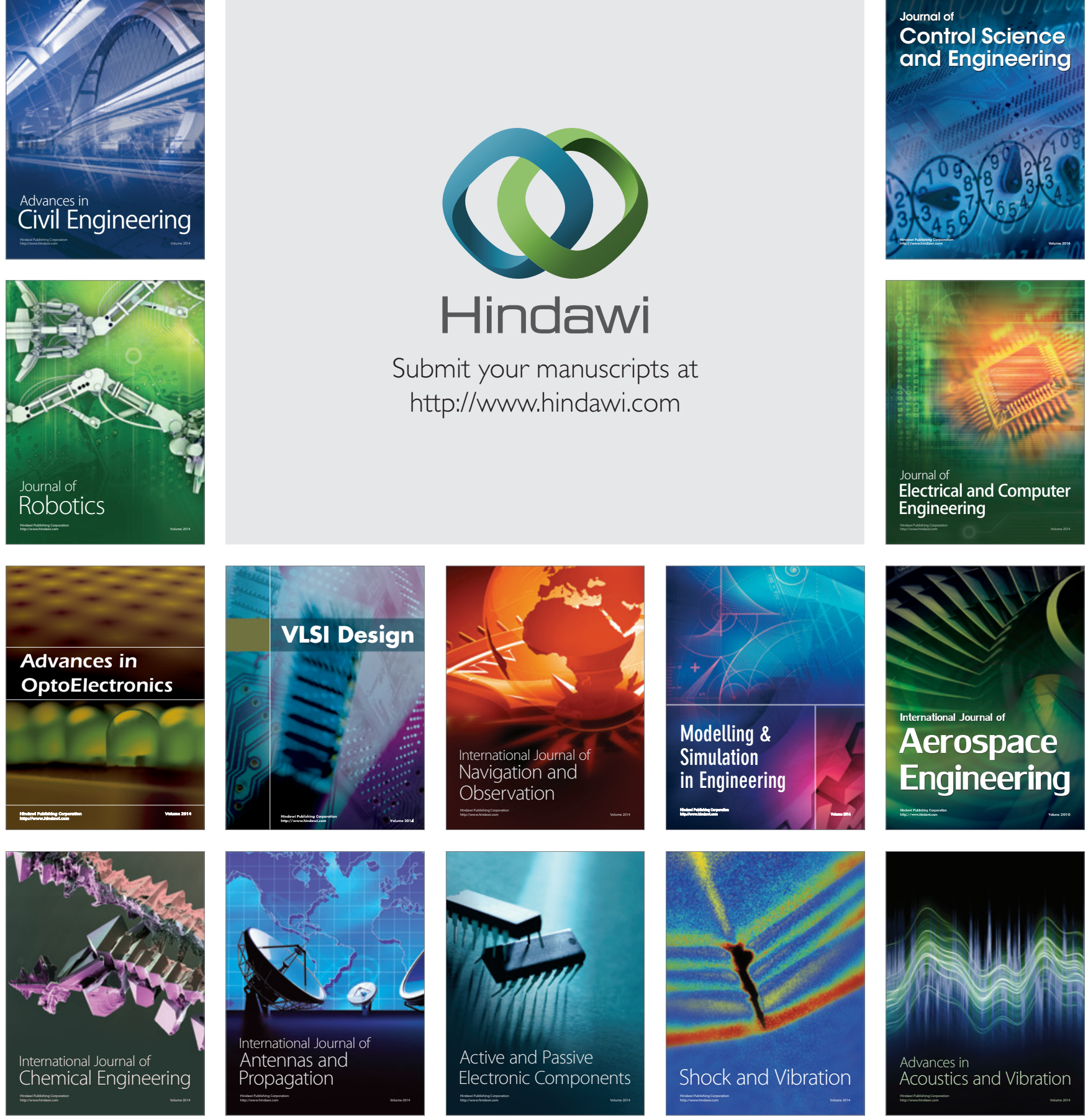